\title{
Aproximaciones metodológicas al estudio de la mortalidad infantil en Chile
}

\author{
R. MAURICIO BARRÍA P. ${ }^{1}$, JAIRO VANEGAS L. ${ }^{2}$ \\ 1. Académico Instituto de Enfermería y Director de la Oficina de Salud Basada en Evidencia (OSBE), Facultad de Medicina, \\ Universidad Austral de Chile. Enfermero, MSc. Epidemiología Clínica, DrPH(c). \\ 2. Programa de Doctorado en Salud Pública, Escuela de Salud Pública, Universidad de Chile. Médico Cirujano, MPH, DrPH.
}

\begin{abstract}
Methodological approaches to the study of infant mortality study in Chile

Introduction: Infant Mortality Rate (IMR) is still considered a relevant population health indicator. Knowing related factors and implementation of specific policies positively impacts its reduction. Objective: To evaluate the evolution of the studies on infant mortality in Chile in the last three decades. Patients and Methods: A systematic search using Medline, Lilacs and Scielo databases was carried out. Global and component IMR was reviewed. Results: IMR has been reduced considerably since 1980. Sixty-eight papers were found, mostly in national journals and bulletins. Most of them (73.5\%) were descriptive studies, although association studies have increased since 2000. The main data source was National Institute of Statistics (47\%). The most frequently studied variables were: cause of death (42.6\%), maternal age (30.9\%) and site (17.6\%). Analysis strategies are mostly descriptive, although studies of association most often utilize correlation indices. Conclusion: Infant Mortality continues to be a topic of interest to researchers, who use different strategies. Continued study is encouraged.
\end{abstract}

(Key words: Infant mortality, epidemiologic research design, demography).

Rev Chil Pediatr 2012; 83 (1): 33-41

\section{RESUMEN}

Introducción: La tasa de mortalidad infantil (TMI) sigue siendo considerada un indicador relevante de salud poblacional. Su estudio ha permitido conocer factores relacionados e implementar políticas específicas que han impactado positivamente en su reducción. Objetivo: Evaluar la evolución de los estudios sobre mortalidad infantil en Chile en las últimas décadas. Pacientes y Método: Se realizó una búsqueda sistemática en las bases referenciales Medline, Lilacs y Scielo. Se contrastó esta información con la TMI global y por componente. Se detectaron 68 artículos publicados en su mayoría en revistas y boletines nacionales. Resultados: Se observó una reducción de la TMI en el período, en tanto las publicaciones del tema aumentaron. Si bien 73,5\% correspondió a estudios descriptivos, desde 2000 los estudios de asociación han aumentado. La principal fuente de datos corresponde al Instituto Nacional de Estadísticas (47\%) y las variables más estudiadas fueron: causas de muerte (42,6\%), edad materna (30,9\%) y localidad (17,6\%). Las estrategias de análisis en su mayoría se basan

Trabajo recibido el 15 de febrero de 2011, devuelto para corregir el 05 de mayo de 2011, segunda versión el 08 de agosto de 2011, aceptado para publicación el 22 de octubre de 2011.

Correspondencia a:

R. Mauricio Barría P.

E-mail: rbarria@uach.cl 
en estadística descriptiva mientras los de asociación lo hacen principalmente mediante análisis de correlación. Conclusión: La mortalidad infantil sigue siendo un tópico de interés para investigadores y para su estudio se han intentado distintas aproximaciones. Los sitios disponibles con información al respecto plantean la instancia de continuar focalizando en el tema para ayudar en su comprensión.

(Palabras clave: Mortalidad infantil, diseño de investigación epidemiológica, demografía).

Rev Chil Pediatr 2012; 83 (1): 33-41

\section{Introducción}

Las políticas de salud se fundamentan en la identificación de áreas problema y el establecimiento de prioridades y alternativas de acción. Las estadísticas vitales sobre mortalidad infantil han sido empleadas ampliamente para establecer el número de muertes infantiles en el primer año de vida de manera global o desagregada y por áreas geográficas, siendo la tasa de mortalidad infantil (TMI) un indicador que resume la calidad de la infraestructura sanitaria, su entorno y como estos pueden actuar negativamente sobre la salud infantil ${ }^{1}$. De esta manera constituye un importante indicador de salud de un país dada su relación con factores como la salud materna, calidad y acceso a servicios médicos, condición socioeconómica y políticas de salud pública. Si bien se ha cuestionado su valor como medida de resumen de salud poblacional ${ }^{2}$, se reconoce que sigue siendo un indicador útil y más fácilmente manejable respecto a otros ${ }^{3,4}$.

Durante el siglo veinte se produjeron notables descensos de la mortalidad en casi todos los países del mundo, independientemente de condiciones socioeconómicas y nivel de desarrollo. En Chile, se ha observado una reducción sostenida de la mortalidad infantil ${ }^{5,6}$, disminuyendo en los últimos 30 años a la décima parte, alcanzando en 2007 una tasa de 8,3 por mil nacidos vivos ${ }^{7}$. Si bien las tasas se asemejan a la de países desarrollados, es posible pensar en abordar causas reducibles para lograr disminuirla más.

Cabe mencionar que el comportamiento de la TMI no siempre es uniforme dentro de un país constatándose que los niveles de reducción no son homogéneos. Esto hace necesario recontextualizar la mortalidad infantil entendiéndola como problema de gestión y planificación local. A este nivel, la TMI es un im- portante indicador para focalizar los recursos y acciones que contribuyan en su reducción. Como ejemplo se cuenta con las metodologías georeferenciales que constituyen una herramienta importante para la epidemiología en procesos de planificación por sectores, aunque su uso en Salud Pública ha sido limitado ${ }^{9}$.

Conocer y analizar tendencias, causas y factores condicionantes de la mortalidad infantil permiten diseñar y fortalecer las estrategias de salud pública destinadas a reducirla ${ }^{10}$. Para esto se han publicado variados estudios sobre mortalidad infantil enfocándose desde distintos puntos de vista (clínico, epidemiológico, demográfico) tratando de explicar tendencias, causas y relaciones con factores específicos. La búsqueda de un mejor entendimiento del fenómeno y el intento por identificar condiciones factibles de intervenir, hace que los estudios adopten distintos diseños metodológicos y estrategias analíticas.

El objetivo de este trabajo es evaluar la evolución de los estudios sobre mortalidad infantil en Chile en las últimas 3 décadas, sus medios de divulgación, los diseños utilizados, los factores o variables estudiadas y las estrategias de su análisis contrastándose con la evolución de la TMI.

\section{Pacientes y Método}

Los datos sobre mortalidad infantil fueron obtenidos desde el sitio web del Departamento de Estadísticas e Información en Salud (DEIS) del Ministerio de Salud de Chile. Para la identificación de artículos se realizó una búsqueda sistemática en Medline, Lilacs, Scielo y ScieloSP. En Medline (a través de Pubmed), se empleó como términos iniciales de búsqueda los descriptores Infant Mortality (MeSH) y el descriptor Chile (MeSH) como Afiliación. 
Adicionalmente se ejecutó una búsqueda con estos términos como palabra de título o del resumen. Se hizo combinación de términos mediante conector AND. En Scielo y Lilacs se empleó estrategia similar usando los descriptores Mortalidad Infantil (descriptor de asunto) y Chile (País), Mortalidad e Infantil como palabras de resumen y como palabras de título y las conexiones entre términos mediante conector AND. Se limitó la búsqueda por año de publicación entre 1980 y 2007. Se limitó a este último año dado que correspondió al último con datos disponibles sobre mortalidad.

Además, se realizó una búsqueda manual selectiva a las revistas chilenas en que por su orientación podrían encontrarse artículos relacionados con Mortalidad Infantil y que eventualmente no hayan estado indexados en las bases. Estas fueron la Revista Chilena de Obstetricia y Ginecología, Revista Chilena de Pediatría, Revista Médica de Chile y Revista Chilena de Salud Pública. Se seleccionó las publicaciones una vez leído los resúmenes y constatándose el abordaje de la problemática específica de Mortalidad Infantil de manera global como evento de interés o de alguno de sus componentes: mortalidad neonatal, neonatal precoz, mortalidad infantil tardía. No se incorporó aquellas publicaciones en las que ésta fue considerada evento secundario o fue mostrada como dato marginal. Tampoco se incluyó aquellas publicaciones como editoriales o comentarios. Bajo estos criterios se identificaron 68 publicaciones editadas entre $1980 \mathrm{y}$ 2007. Se accedió a todos los artículos a texto completo en versión impresa o en línea. Se clasificaron las publicaciones por revista, año de publicación, diseño metodológico, tipo de análisis y principales variables de estudio. No se excluyó ninguna de las publicaciones seleccionadas inicialmente bajo criterios o juicio de calidad dado que la intención del trabajo fue mostrar el abordaje del fenómeno de interés y como ha sido la aproximación metodológica. En consecuencia, este estudio no persiguió evaluar la pertinencia y aplicabilidad de los diseños y el manejo estadístico de la información, sino más bien, exponer como se ha estudiado.

El diseño metodológico fue considerado descriptivo cuando se mostró sólo la evolución, tendencia y la distribución de causas y factores relacionados con la mortalidad. En este fueron considerados los reportes demográficos y las series de tiempo. Se consideró diseño de asociación cuando el trabajo relacionó factores específicos con la mortalidad infantil general o por sus componentes empleando diseños ecológicos o de nivel individual.

Se identificaron las principales variables estudiadas en las distintas publicaciones focalizando en aquellas que buscaron explicar o evaluar el comportamiento de la mortalidad.

Para evaluar el tipo de estrategia analítica se dividieron en uso de distribución de frecuencias, comparación de grupos usando inferencia estadística a través de test de $\chi^{2}$, exacto de Fisher, estimación de riesgo, correlación de Spearman o Pearson y/o modelación (Poisson, Logística).

\section{Resultados}

La TMI evidenció un descenso significativo desde 33 por mil nacidos vivos en 1980 a 8,3 en 2007 mostrando una reducción relativa de $74,8 \%$. Este descenso ha sido diferencial de acuerdo a los componentes de la TMI. La mortalidad neonatal se redujo en $66,5 \%$ mientras la mortalidad postneonatal lo ha hecho en $83,4 \%$ en este período.

Los 68 artículos identificados se distribuyeron de manera similar en las dos primeras décadas estudiadas, registrándose 20 entre 19801989 y 19 entre 1990-1999. Se evidenció un incremento a partir del año 2000 totalizando a 2007 veintinueve publicaciones, mostrando un aumento relativo de 52,6\% respecto a la década precedente.

Respecto del número de artículos publicados por año, destaca en 1982 que fueron publicados 8 trabajos (40\% del total de la década). En los últimos 7 años de estudio la producción de ellos ha sido más constante, observándose entre 2000 y 2004 un número no inferior a tres por año, contrastando con la desaceleración de la reducción de la TMI (figura 1).

Las revistas que más han publicado trabajos de este tipo son la Revista Chilena de Pe- 
diatría, Revista Chilena de Obstetricia y Ginecología y la Revista Médica de Chile con 30,9, 17,6 y $14,7 \%$ respectivamente. Se detectaron $11(16,2 \%)$ publicaciones en revistas extranjeras (tabla 1 ).

El 73,5\% de los artículos fueron considerados estudios descriptivos mostrando series temporales, tendencias de mortalidad tanto global como por componentes, estudios de enfoque demográfico y experiencias clínicas y de servicios de salud específicos. En 18 trabajos (26,5\%) la propuesta fue analítica evaluándose asociación o correlación con factores clínicos, factores sociodemográficos y socioeconómicos. Dentro de estos últimos se encontraron dos trabajos especificados como estudio ecológico y un artículo que presenta modelación y simulación estadística.

La tendencia ha sido inversa entre los estudios descriptivos y aquellos que buscan establecer asociación o correlación. Así se observa que en la década del 80 las publicaciones eran eminentemente descriptivas para luego en la década siguiente ir dando cabida a los estudios de asociación que finalmente a partir del año 2000 ya alcanzan $44,8 \%$ (figura 2 ).

La principal fuente de datos sobre mortalidad infantil corresponde al Instituto Nacional de Estadísticas (INE). En 28 artículos (41,2\%) se cita como fuente de información el Anuario de Demografía del INE. No obstante, el INE es mencionado como fuente de datos en $47 \%$ de las publicaciones analizadas. Por su parte, $45,5 \%$ de los trabajos presentan mortalidad por causas específicas las que son clasificadas de acuerdo a la Clasificación Internacional de Enfermedades (CIE) en sus diferentes versiones. Otras fuentes de datos consignadas en lo estudios son bases e informes del Ministerio de Salud y la información del registro civil. Los estudios sobre población hospitalaria se basan en expedientes médicos o fichas clínicas y auditorías.

El análisis de los datos y su presentación está basado fundamentalmente en tablas y gráficos de distribución de frecuencias y de tendencia de la mortalidad. Tres trabajos presentan medidas de tendencia central (promedio). En menos casos se ha empleado inferencia estadística y pruebas de hipótesis. El uso de test $\chi^{2}$ o exacto de Fisher se especificó en 6 estudios. Tres trabajos presentaron análisis en base a t-test. El empleo de análisis de correlación de Pearson y Spearman, fue mencionado en 9 y 2 publicaciones respectivamente. Mientras la estimación de riesgo en base a odds ratio, riesgo relativo o riesgo atribuible fue mencionada en 7 trabajos. En una publicación se presentó análisis estratificado y un modelo de regresión logística mientras en otro un modelo Poisson.

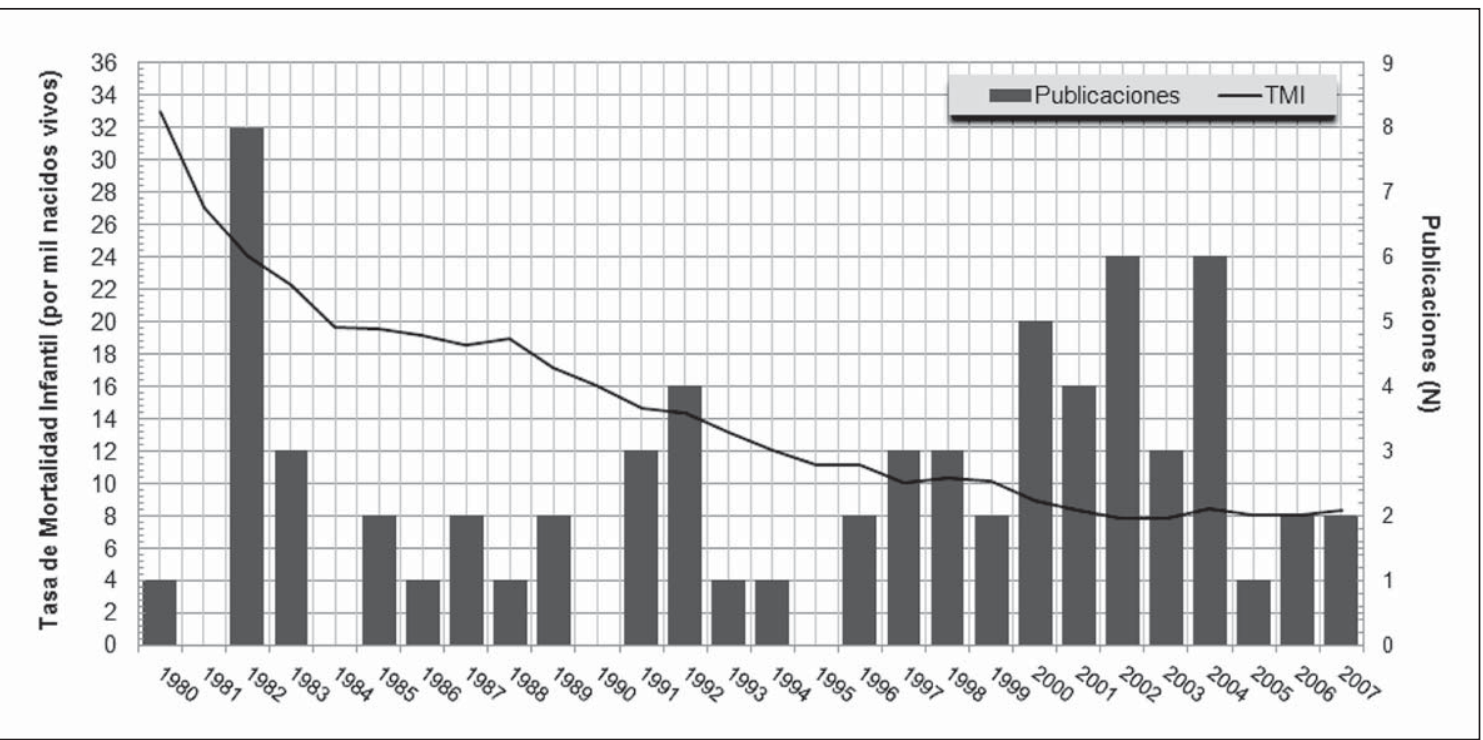

Figura 1. Tasa de Mortalidad Infantil y publicaciones por año período 1980 - 2007. TMI: tasa de mortalidad infantil. 
Tabla 1. Número de publicaciones sobre mortalidad infantil en Chile por año y revista

\begin{tabular}{|c|c|c|c|c|c|}
\hline \multirow[t]{2}{*}{ Revista } & \multicolumn{3}{|c|}{ Publicaciones por período } & \multicolumn{2}{|c|}{ Total } \\
\hline & 1980-1989 & 1990-1999 & $2000-2007$ & $\mathbf{n}$ & $\%$ \\
\hline Rev Chil Pediatr & 7 & 7 & 7 & 21 & 30,9 \\
\hline Rev Chil Obst Ginecol & 2 & 3 & 7 & 12 & 17,6 \\
\hline Rev Med Chile & 2 & 3 & 5 & 10 & 14,7 \\
\hline Bol Oficina Sanit Panam & 3 & 0 & 0 & 3 & 4,4 \\
\hline Pediatría (Santiago) & 1 & 1 & 0 & 2 & 2,9 \\
\hline Bol Epidem Chile & 2 & 0 & 0 & 2 & 2,9 \\
\hline Cuad Méd Soc (Chile) & 0 & 1 & 1 & 2 & 2,9 \\
\hline Rev Chil Enferm Respir & 0 & 2 & 0 & 2 & 2,9 \\
\hline Rev Hosp Clin U de Chile & 0 & 1 & 1 & 2 & 2,9 \\
\hline Am J Public Health & 1 & 0 & 0 & 1 & 1,5 \\
\hline Anales de Pediatría & 0 & 0 & 1 & 1 & 1,5 \\
\hline Applied Economics & 0 & 1 & 0 & 1 & 1,5 \\
\hline Bol Hosp San Juan de Dios & 1 & 0 & 0 & 1 & 1,5 \\
\hline Bull World Health Organ & 0 & 0 & 1 & 1 & 1,5 \\
\hline Environ Health Perspect & 0 & 0 & 1 & 1 & 1,5 \\
\hline J Perinat Med & 1 & 0 & 0 & 1 & 1,5 \\
\hline Pediatr día & 0 & 0 & 1 & 1 & 1,5 \\
\hline Pediatrics & 0 & 0 & 1 & 1 & 1,5 \\
\hline Rev Chil Salud Pública & 0 & 0 & 1 & 1 & 1,5 \\
\hline Rev Med del Maule & 0 & 0 & 1 & 1 & 1,5 \\
\hline Rev Panamericana Salud Pub & 0 & 0 & 1 & 1 & 1,5 \\
\hline Total & 20 & 19 & 29 & 68 & 100 \\
\hline
\end{tabular}

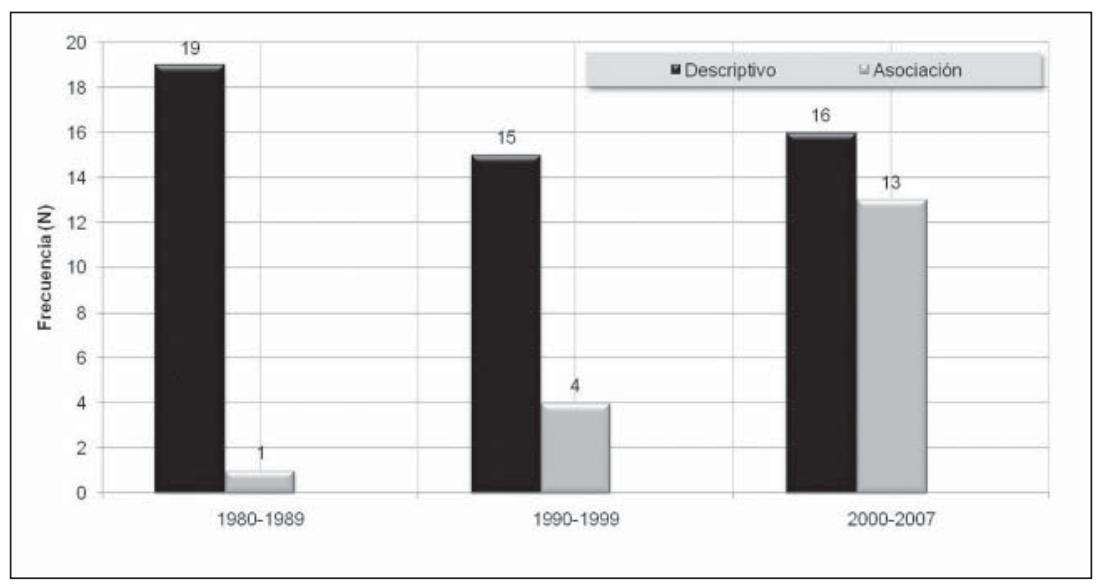

Figura 2. Diseños metodológicos en el estudio de la Mortalidad Infantil en Chile. 
Es necesario aclarar que algunos trabajos incluyeron más de una de las categorías clasificadas y por ende, se muestra la frecuencia en que cada categoría fue incluida en los diferentes estudios (tabla 2).

Las variables más estudiadas corresponden a las causas o diagnósticos de muerte. La edad materna por su parte, es un factor clave que ha sido estudiado y vinculado a la mortalidad infantil. Otros factores importantes considerados en los estudios son la educación materna y el orden de nacimiento. Desde el punto de vista social, el estado civil, el nivel socioeconómico y la legitimidad del niño son otras variables de importancia. Por último, factores del entorno como la atención médica y el contexto económico también han sido estudiados enfatizándose en la última década (tabla 3).

\section{Discusión}

Las estadísticas vitales siguen siendo fundamentales para el análisis de situaciones actuales de salud, como indicador de resultado de intervenciones y de implementación de políticas específicas. La posibilidad de seguir estudiando el tema y la apertura de las revistas a publicar trabajos de este tipo y mantener espacios para reflexiones sobre salud pública, potencian el interés de investigadores a profundizar en el área.

Los estudios sobre mortalidad infantil tradicionalmente se clasifican en investigaciones con énfasis demográfico y las de enfoque epidemiológico. Se consideran también aquellas que destacan factores sociales como variable principal en el proceso de explicación o interpretación del proceso de mortalidad ${ }^{11}$. Los estudios demográficos describen tendencias de la mortalidad infantil, su variación, las relaciones internas entre sus componentes y la comparación por áreas geográficas. Por su parte, la epidemiología y sus métodos buscan establecer relaciones de la mortalidad infantil con variables biológicas, ambientales, económicas y sociales. Esto es fundamental dado que se deben considerar en el estudio de la mortalidad infantil el conjunto de factores que influyen y determinan su nivel.

La influencia de estos factores difiere según
Tabla 2. Estrategias de análisis descritas en las 68 publicaciones

\begin{tabular}{|lrr|}
\hline $\begin{array}{l}\text { Análisis - Presentación de } \\
\text { Resultados }\end{array}$ & n & \% \\
\hline $\begin{array}{l}\text { Tendencia - Evolución TMI o por componente } \\
\text { Distribución de frecuencias por causa o por }\end{array}$ & 33 & 30,9 \\
componente de Ml & 2 & 2,9 \\
\hline Medidas de Tendencia Central (Promedio) & 3 & 4,4 \\
\hline T - Student & 6 & 8,8 \\
\hline Test $\chi^{2}$ o Exacto de Fisher & 11 & 16,2 \\
\hline Correlación Pearson - Spearman & 7 & 10,3 \\
\hline Riesgo (Rate ratio; Riesgo Relativo; Odds Ratio) & 2 & 2,9 \\
\hline Modelo de Regresión (Logística- Poisson) & 1 & 1,5 \\
\hline Modelo multiecuación/Simulación & & 21 \\
\hline
\end{tabular}

TMI: tasa de mortalidad infantil; MI: mortalidad infantil. *Nota: hay estudios que consideran más de una alternativa por lo cual la sumatoria es mayor a $100 \%$ y 68 publicaciones.

Tabla 3. Principales factores o variables estudiadas en los 68 artículos

\begin{tabular}{|lcc|}
\hline Variable & n & \% \\
\hline Causas/Diagnóstico de muerte & 29 & 42,6 \\
\hline Edad materna & 21 & 30,9 \\
\hline Localidad: región - comuna & 12 & 17,6 \\
\hline Peso de Nacimiento & 12 & 17,6 \\
\hline Educación materna & 11 & 16,2 \\
\hline Orden de nacimiento & 9 & 13,2 \\
\hline Sexo & 6 & 8,8 \\
\hline Nivel socioeconómico & 5 & 7,3 \\
\hline Estado Civil & 5 & 7,3 \\
\hline Edad gestacional & 6 & 8,8 \\
\hline Atención médica & 4 & 5,9 \\
\hline Legitimidad & 4 & 5,9 \\
\hline $\begin{array}{l}\text { Variables económicas: PIB - Ingreso per } \\
\text { cápita, etc. }\end{array}$ & 4 & 5,9 \\
\hline *Nota: hay estudios que consideran más de una alternativa por lo \\
Cual la sumatoria es mayor a 100\% y 68 publicaciones.
\end{tabular}

la edad de los menores de un año y, por ende, cada componente de la mortalidad infantil obedece a etiologías diferentes. En este sentido, usar tasas globales de mortalidad infantil para determinar la eficacia de intervenciones no ayuda a las comunidades a centrarse en factores subyacentes particulares que contri- 
buyen a su estancamiento o desaceleración ${ }^{12}$. En la mortalidad neonatal prevalecen factores vinculados con condiciones congénitas y con la atención de salud como el control prenatal, acceso y atención profesional.

En la mortalidad postneonatal tiene mayor impacto las condiciones ambientales y socioeconómicas sobre la salud del niño. Pareciera ser que las muertes por factores exógenos pueden ser reducidas más rápidamente por intervenciones sanitarias, lo que se ha manifestado en los grandes descensos de la TMI. No obstante, la desaceleración y la baja diferencial en sus componentes suponen considerar las causas de muerte neonatal las que pueden ser más complejas de prevenir.

El origen de la mayoría de las muertes neonatales está relacionado a complicaciones durante el embarazo, como hemorragias, sepsis, eclampsia así como partos complicados, por lo que están íntimamente relacionadas a la mortalidad materna.

Aunque Chile ha reducido su TMI en las últimas tres décadas hasta niveles de países desarrollados, es posible pensar que esto aún podría mejorar. Estudios recientes establecen que parte de la TMI está relacionada a madres sin educación, observándose un mayor riesgo por enfermedades de origen infeccioso y traumatismos. También, se destaca un mayor riesgo según niveles socioeconómicos, origen étnico así como inequidad en la cobertura de las estadísticas vitales. Esto afirma que las clases sociales desfavorecidas están expuestas a una mayor mortalidad infantil por infecciones y traumatismos ${ }^{13}$.

Consecuentemente, se requiere que la focalización sea mayor en la búsqueda de un factor o un grupo de ellos que puedan ser abordados. La posibilidad de emplear nuevas estrategias de aproximación al fenómeno de la mortalidad infantil plantea importantes desafíos a los investigadores. Los estudios sobre mortalidad infantil han tratado de dimensionar las causas de diferencias en la mortalidad dando a conocer la gama de factores y relaciones complejas que reflejan la interacción de factores socioeconómicos, ambientales, culturales y étnicos.

Desde el punto de vista metodológico, una particularidad observada en los estudios es la posibilidad de que la mayoría de las variables tengan limitaciones de carácter estadístico tales como la multicolinealidad o la no independencia entre las variables debido a la fuerte asociación que presentan algunos factores relacionados con la TMI. La multicolinealidad no obstante, no siempre puede considerarse un problema, por ejemplo, si el objetivo es solamente pronóstico, donde mientras más alto sea el $\mathrm{R}^{2}$ mejor será la predicción. De tal forma, lo importante de la colinealidad, no es su presencia, sino el grado de perturbación que puede introducir a un modelo. Si el objetivo es asociar, habrá que analizar el grado de colinealidad existente para no incurrir en posibles asociaciones espurias, tratando de obtener modelos lo más plausibles posibles. En consecuencia, la multicolinealidad es un problema de grado y no de clase. Pero, por lo general, este aspecto poco se menciona, lo cual es relevante para las estimaciones. También es importante la exploración de interacciones así como la detección de posibles factores de confusión para poder obtener aproximaciones más adecuadas.

En el caso de las series de tiempo, a veces no se menciona si hubo exploración de aspectos como la estacionalidad de las variables. Esto quiere decir que el valor de las medias y su varianza no deberían variar sistemáticamente en el tiempo seleccionado para su estudio. La importancia de este análisis radica en la posibilidad de generar asociaciones espurias, debido a que las asociaciones encontradas podrían obedecer más a las fuertes tendencias debido a la declinación de los valores y no a una asociación real. Otro aspecto no menos significativo es la consideración de los retardo, en particular cuando algunas variables están relacionadas al ámbito de lo económico. La dependencia de una variable "Y" respecto de otra $\mathrm{u}$ otras variables " $\mathrm{X}$ ” pocas veces tiene efecto inmediato. Es frecuente que dicho efecto se dé en un lapso de tiempo determinado, o sea, que el efecto sobre Y ocurra mucho después.

En este sentido existe controversia en el ámbito académico sobre cuando rezagar una variable económica sobre la mortalidad infantil. Algunos plantean cuatro años de rezago, sin embargo, al respecto no hay consenso ya que mientras más rezagos se incluyen se pierden 
grados de libertad haciendo que la inferencia estadística se vaya debilitando y por otra parte, los rezagos sucesivos pueden acompañarse de alta correlación y por tanto, conducir a colinealidad. Estas limitaciones metodológicas invitan a considerar modelaciones de carácter no lineal.

Si bien podría pensarse que todo niño que nace posee igual riesgo de morir, esta probabilidad claramente varía de acuerdo con las condiciones y factores de riesgo presentes. En este contexto, aparentes asociaciones entre factores específicos y la mortalidad infantil pueden deberse a la acción de factores adicionales lo cual es habitual cuando se estudia factores sociales y demográficos en que los efectos estimados son relativamente pequeños y donde la incorporación de confundentes en el análisis tiende a distorsionar una relación, pudiendo incrementar, reducir, revertir o anular una asociación. Lo anterior sólo muestra lo importante de dar soluciones a estos aspectos inferenciales para lograr una mejor aproximación y obtener mejores interpretaciones.

El peso que tiene cada factor o variable sobre la reducción mostrada por la mortalidad infantil es difícil de determinar dada la ausencia de datos necesarios y por correlación entre ellos ${ }^{14}$. Este es otro problema de primer orden relacionado a la calidad de los datos, ello implica mejorar los registros y capacitar al personal sanitario en la completación de datos y diagnósticos, reduciendo de esta manera la información mal clasificada o imprecisa. El manejo de las variables, sus niveles de medición y las estrategias analíticas son fundamentales para establecer modelos explicativos de la mortalidad infantil basados, por ejemplo, en el peso y la edad gestacional ${ }^{15}$. Así, el uso de análisis multivariante, como los modelos no lineales, puede ayudar a los investigadores a controlar o reducir el efecto de estos factores. No obstante, el conocimiento de relaciones básicas entre variables y factores específicos con la mortalidad infantil son precursores necesarios para análisis más sofisticados.

Como se mencionó, este trabajo intentó recopilar la producción científica y divulgación de estudios sobre un tema fundamental como indicador de salud, cual es la mortalidad in- fantil, y describir los elementos involucrados en su estudio, en especial, los factores que han sido considerados en su explicación. Consecuentemente, debemos asumir que existen limitantes posibles al no incluir trabajos que no llegaron a ser publicados o cuyos datos se encuentran formando parte de informes técnicos o similares. De esa forma, un cuestionamiento válido es interrogarse si -así como ocurre en otras áreas-, todo lo que realmente se estudia se difunde o publica, lo que comportaría un sesgo de publicación. Otra limitación de este trabajo es que no se realizó un análisis de la pertinencia de los diseños y las técnicas estadísticas empleadas dado que como se comentó, el propósito fue mostrar la forma en que los investigadores se han aproximado al fenómeno en cuestión.

Como hallazgos de interés puede mencionarse que el tema de la mortalidad infantil es un tópico de investigación vigente y se dispone de sitios en los cuales es posible obtener información agregada o desagregada para realizar estudios futuros sobre la mortalidad y como complementarla con información relevante de índole social o económica también disponible.

\section{Referencias}

1.- Donoso E: Desigualdad en mortalidad infantil entre las comunas de la provincia de Santiago. Rev Med Chile 2004; 132 (4): 461-6.

2.- Mathers CD, Salomon JA, Murray CJ: Infant mortality is not an adequate summary measure of population health. J Epidemiol Community Health 2003; 57 (5): 319.

3.- Reidpath $D D$, Allotey P: Infant mortality rate as an indicator of population health. J Epidemiol Community Health 2003; 57 (5): 344-6.

4.- Robine JM: The relevance of population health indicators. J Epidemiol Community Health 2003; 57 (5): 318.

5.- Águila A, Muñoz H: Tendencia de la Natalidad, Mortalidad General, Infantil y Neonatal en Chile desde el año 1850 a la Fecha. Rev Med Chile 1997; 125 (10): 1236-45.

6.- Szot J: Mortalidad Infantil en Chile: 1989-1998. Rev Chil Pediatr 2002; 73 (2): 164-6.

7.- Szot J. Disminución de la mortalidad infantil en Chile entre 1990 y 2001. An Pediatr (Barc) 2004; 61 (3): 22630. 
8.- Departamento de Estadísticas e Información de Salud (DEIS): Mortalidad Infantil y sus componentes por región y comuna, 2007. [Disponible en: URL: http://163.247.51.54/nuev/salida/tab06_07.php] [Consulta: 10 de diciembre de 2009].

9.- Spinelli H, Alazraqui M, Calvelo L, Arakaki J: Mortalidad Infantil “Un indicador de Gestión Local” Análisis de mortalidad infantil de las provincias de Buenos Aires en 1998. Organización Panamericana de la Salud Publicación No 51 Buenos Aires 2000.

10.- Kaempffer AM, Medina E: Análisis de la mortalidad infantil y factores condicionantes. Chile, 1998. Rev Chil Pediatr 2000; 71 (5): 405-12.

11.- Troncoso M: Mortalidad infantil: revisando un indicador de salud. En: Duarte E, editor. Ciencias sociales y salud en la América Latina, tendencias y perspectivas. OPSCIESU; 1986.

12.- Burns $P G$ : Reducing infant mortality rates using the perinatal periods of risk model. Public Health Nurs 2005; 22 (1): 2-7.

13.- Hertel A, Giusti A, Sotelo J: The Chilean infant mortality decline: improvement for whom? Socioeconomic and geographic inequalities in infant mortality 1990-2005. Bull World Health Organ 2007; 85 (10): 798-804.

14.- Taucher E, Jofré I: Mortalidad infantil en Chile: el gran descenso. Rev Med Chile 1997; 125 (10): 1225-35.

15.- Solis P, Pullum SG, Frisbie WP: Demographic models of birth outcomes and infant mortality: an alternative measurement approach. Demography 2000; 37 (4): 48998. 